Ann. Sci. forest., 1979, 36 (2), 151-164.

\title{
Fertilisation minérale et fructification des champignons supérieurs en hêtraie
}

\author{
J. GARBAYE, A. KABRE, F. LE TACON, D. MOUSAIN * \\ et D. PIOU \\ Station de Recherches sur les Sols forestiers ef la Fertilisation \\ Centre national de Recherches forestières, I.N.R.A., \\ Champenoux, 54280 Seichamps \\ * Laboratoire de Recherches sur les Symbiotes des racines, I.N.R.A.-E.N.S.A., \\ 9, place Viala, 34060 Montpellier Cedex
}

\section{Résumé}

Tous les carpophores des champignons supérieurs visibles début octobre 1977 ont été comptés dans un essai de fertilisation sur hêtre (Fagus silvatica) de 90 ans dans la région des Basses Vosges. Le sol développé sur grès à voltzia (Trias inférieur), varie du sol brun acide à mull acide au sol brun ocreux à moder. Sur les cinquante-trois espèces inventoriées, quinze étaient assez abondantes pour faire l'objet d'une interprétation en fonction de la fertilisation et du type d'humus. Elles peuvent être classées en quatre groupes selon leur comportement :

- espèces réagissant essentiellement à l'apport de calcaire (type : Lycoperdon gemmatum, qui n'est présente que sur les traitements comprenant une forte dose de calcaire seul) ;

- espèces réagissant essentiellement à l'apport de phosphore (type : Boletus chrysenteron, dont la fructification est favorisée par le phosphore et contrariée par le calcium);

— espèces réagissant essentiellement à la fertilisation complète NPKCa (type : Lepista inversa, qui n'apparaît que sur les traitements complets) ;

- espèces réagissant essentiellement au type d'humus (type : Russula fellea, qui n'apparaît que sur les mull-moder et surtout sur les moder).

Enfin, la fertilisation semble présenter un intérêt économique pour une espèce comestible : Rhodopaxillus nudus (Tricholome nu, pied bleu), qui est totalement absent dans les témoins, alors que $58 \mathrm{~kg}$ de carpophores par hectare ont été récoltés sur le traitement complet NPKCa en un mois. Un rapide calcul montre que la valeur des champignons produits annuellement est au moins égale à celle du bois, et qu'elle peut rembourser en 2 ou 3 ans la dépense occasionnée par la fertilisation.

\section{Introduction}

Les conditions édaphiques de la fructification des champignons supérieurs sont encore très mal connues. Quelques travaux remarquables ont cependant été publiés en Suisse, en Belgique et Allemagne. Ainsi, Kraft a étudié la répartition en Suisse d'Amanifa caesarea (1956) et celle d'Hygrophorus marzuolus (1958). Darimont a effectué l'analyse mycosociologique des formations silvatiques caducifoliées de Haute-Belgique (1973). Concernant l'Allemagne, citons les travaux de Einhellinger (1964) sur 
les chênaies-charmaies de la région de Munich, et la synthèse de Jahn, Nespiak et Tuxen (1967).

En France, citons pour la région de l'Est l'excellente étude de Carbiener, Ourisson et Bernard réalisée dans la plaine d'Alsace, et une approche de Chevassut et Mousain (1973) relative à la mycosociologie des peuplements de Pin maritime dans la région de Montpellier.

Ces travaux montrent que les champignons supérieurs réagissent avec une très grande sensibilité aux variations de milieu et qu'ils peuvent constituer d'excellents indicateurs stationnels ou microstationnels.

Nous avons utilisé un dispositif de fertilisation installé depuis six ans dans une hêtraie des Basses Vosges pour tenter de préciser expérimentalement certaines conditions édaphiques de la fructification de quelques champignons supérieurs saprophytes ou mycorhiziens.

\section{Matériel et méthodes}

\section{Les conditions de milieu}

La forêt de Darney où est installé le dispositif est située dans les Basses Vosges, entre 300 et $400 \mathrm{~m}$ d'altitude. La pluviosité est de $950 \mathrm{~mm}$, assez régulièrement répartie avec un léger maximum en juillet-août et un léger minimum en janvier-février-mars. La température moyenne de juillet est de $18^{\circ}$ et celle de janvier de $-1,7^{\circ}$.

La forêt de Darney s'étend sur le trias, du grès vosgien au Muschelkalk moyen. La hêtraie correspond essentiellement aux affleurements du grès à voltzia et du grès intermédiaire.

Sur grès à voltzia, les sols sont le plus souvent des sols bruns acides à mull acide, plus rarement à mull-moder, ou des sols bruns ocreux à moder.

Les sols bruns acides à mull acide sont caractéristiques de la hêtraie à Luzule blanche (Luzula albida).

Les sols bruns acides à mull-moder et bruns ocreux à moder sont caractéristiques de la hêtraie à Canche flexueuse (Deschampsia flexuosa).

Ces deux types de hêtraies sont parmi les plus répandus dans les Basses-Vosges. Dans le dispositif de fertilisation, ces deux types de hêtraie sont représentés (tabl. 1 et 2).

Les réserves en eau utile de ces deux profils sont assez importantes en raison de la charge non négligeable en limons et en argile et en raison de l'épaisseur du profil (1 m au moins).

\section{Le peuplement}

Le peuplement est issu d'une régénération naturelle. II s'agit d'une futaie de Hêtre (Fagus silvatica L.) âgée de 90 ans ( \pm 10 ans), en mélange avec quelques chênes rouvres (Quercus petraea (M.) Liebl.) (environ 10 p. 100). D'après la table de production-éclaircie forte de Schober (1972), qui peut être utilisée dans le NE de la France, le peuplement se situe en moyenne dans la première classe de fertilité. 
TABLEAU 1

Hêtraie à luzule, sol brun acide à mull acide sur grès à voltzia avec légère "pollution 》 limoneuse

(Forêt domaniale de Darney)

Soil analysis of a beech site with Luzula albida at Darney ;

acid brown soil, with acid mull on sandstone, slightly covered with eolian loam

\begin{tabular}{|c|c|c|c|c|c|c|c|c|c|c|c|}
\hline & \multirow{2}{*}{$\begin{array}{l}\text { m. o. } \\
\text { p. } 100\end{array}$} & \multirow{2}{*}{$\begin{array}{c}\text { p. } 100 \\
\text { p. }\end{array}$} & \multirow{2}{*}{$\begin{array}{l}N \\
\text { p. } 100\end{array}$} & \multirow{2}{*}{$C / N$} & \multirow{2}{*}{$\mathrm{pH}$} & \multicolumn{4}{|c|}{ m. e. $100 \mathrm{~g}$} & \multirow{2}{*}{$\mathrm{S} / \mathrm{T}$} & \multirow{2}{*}{$\begin{array}{c}\mathrm{P}_{2} \mathrm{O}_{5} \\
\text { p. } 1000\end{array}$} \\
\hline & & & & & & $\mathrm{Ca}$ & $\mathrm{K}$ & $\mathrm{Mg}$ & $\mathrm{T}$ & & \\
\hline $\begin{array}{l}A_{1} 0-5 \ldots \ldots \ldots \\
\text { B } 20-30 \ldots \ldots \\
\text { (B) } 50-60 \ldots \ldots \\
\text { (B) } / C 90-100 \ldots\end{array}$ & $\begin{array}{l}8,1 \\
1,1\end{array}$ & $\begin{array}{l}4,7 \\
0,65\end{array}$ & $\begin{array}{l}0,25 \\
0,06\end{array}$ & $\begin{array}{l}19,0 \\
11,0\end{array}$ & $\begin{array}{l}4,2 \\
4,2 \\
4,2 \\
4,3\end{array}$ & $\begin{array}{l}1,33 \\
0,35 \\
0,23 \\
0,30\end{array}$ & $\begin{array}{l}0,95 \\
0,15 \\
0,15 \\
0,30\end{array}$ & $\begin{array}{l}0,24 \\
0,15 \\
0,05 \\
0,08\end{array}$ & $\begin{array}{r}16,9 \\
6,2 \\
5,6 \\
7,4\end{array}$ & $\begin{array}{r}17,5 \\
12,0 \\
9,5 \\
7,1\end{array}$ & $\begin{array}{l}0,06 \\
0,08 \\
0,08 \\
0,07\end{array}$ \\
\hline
\end{tabular}

Texture sablo-limoneuse. Taux de saturation inférieur à 20 p. 100 en $A_{1}$ et à 10 p. 100 à partir de $50 \mathrm{~cm}$. C/N inférieur à 20. Teneur en phosphore moyenne à faible.

\section{TABLEAU 2}

Hêtraie d̀ canche flexueuse. Sol brun ocreux à moder sur grès (transition grès à Voltzia grès intermédiaire) (Forêt domaniale de Darney)

Soil analysis of a beech site with Deschampsia flexuosa at Darney : acid ochreous brown soil with moder on sandstone

\begin{tabular}{|c|c|c|c|c|c|c|c|c|c|c|c|}
\hline & \multirow{2}{*}{$\begin{array}{l}\text { m. o. } \\
\text { p. } 100\end{array}$} & \multirow{2}{*}{ p. 100} & \multirow{2}{*}{$\begin{array}{c}N \\
\text { p. } 100\end{array}$} & \multirow{2}{*}{$C / N$} & \multirow{2}{*}{$\mathrm{pH}$} & \multicolumn{4}{|c|}{ m. e. $100 \mathrm{~g}$} & \multirow{2}{*}{$\mathrm{S} / \mathrm{T}$} & \multirow{2}{*}{$\begin{array}{r}\mathrm{P}_{2} \mathrm{O}_{5} \\
\text { p. } 1000\end{array}$} \\
\hline & & & & & & $\mathrm{Ca}$ & $\mathrm{K}$ & $\mathrm{Mg}$ & $\mathrm{T}$ & & \\
\hline $\begin{array}{l}\mathrm{A}_{2} 2-5 \ldots \ldots \\
\text { (B) } 10-20 \ldots \ldots \\
\text { (B) } 40-50 \ldots \ldots \\
\text { (B) } / C 80-90 \ldots\end{array}$ & 22,4 & 11,4 & 0,40 & 28,0 & $\begin{array}{l}3,5 \\
4,1 \\
4,1 \\
4,3\end{array}$ & $\begin{array}{l}0,40 \\
0,15 \\
0,15 \\
0,30\end{array}$ & $\begin{array}{l}0,40 \\
0,19 \\
0,15 \\
0,34\end{array}$ & $\begin{array}{l}0,38 \\
0,08 \\
0,06 \\
0,10\end{array}$ & $\begin{array}{r}23,1 \\
10,2 \\
8,7\end{array}$ & $\begin{array}{l}7,5 \\
4,7 \\
9,1\end{array}$ & $\begin{array}{l}0,04 \\
0,04 \\
0,04 \\
0,04\end{array}$ \\
\hline
\end{tabular}

Texture sablo-limoneuse. Taux de saturation très faible inférieur à 10 p. 100 dans tout le profil. C/N élevé. Teneur en phosphore faible.

La hauteur dominante est en moyenne de $29,8 \mathrm{~m}$; elle varie cependant de $25,0 \mathrm{~m}$ à $32,9 \mathrm{~m}$. Cette variation est due à des différences d'âge pratiquement incontrôlables, et aux conditions de station (łypes de sol et d'humus, épaisseur des limons, etc...) qui ne sont pas homogènes sur l'ensemble du dispositif.

Ainsi, un certain nombre de placeaux correspond à la $2^{\mathrm{e}}$ classe de fertilité de la table de production. Le tableau 3 donne les principales caractéristiques du peuplement de l'essai de Darney et les compare aux classes 1 et 2 de la table de production de Schober. 
TABLEAU 3

Comparaison des principales caractéristiques du peuplement de Darney avec les classes 1 et 2 de la table de production de Schober (éclaircie forte)

Principal stand characteristics of the beech stand in Darney

\begin{tabular}{|c|c|c|c|c|c|c|c|c|c|}
\hline \multirow[b]{3}{*}{$\begin{array}{l}\text { Age } \ldots \ldots \ldots \\
\text { Hauteur dominante } \ldots \ldots \ldots \\
\text { Nbre total des tiges/ha...... } \\
\text { Surface terrière totale/ha } \ldots \\
\text { Circonférence moyenne } \ldots . \\
\text { Volume B. F. sur pied/ha } \ldots . \\
\text { Accroissement courant/ha } \ldots \\
\text { Accroissement moyen/ha .... }\end{array}$} & \multicolumn{3}{|c|}{$\begin{array}{l}\text { Peuplement } \\
\text { de Darney }\end{array}$} & \multicolumn{6}{|c|}{$\begin{array}{l}\text { Table de production. } \\
\text { Eclaircie forte (Schober) }\end{array}$} \\
\hline & Min. & $\begin{array}{c}\text { Moy. } \\
90 \\
298\end{array}$ & $\begin{array}{l}\text { Max. } \\
-\overline{329}\end{array}$ & \multicolumn{4}{|c|}{ Classe 1} & \multicolumn{2}{|c|}{ Classe 2} \\
\hline & $\begin{array}{l}-\overline{25,0} \\
294 \\
24,6 \\
69,0 \\
- \\
-\end{array}$ & $\begin{array}{l}90 \\
29,8 \\
571 \\
32,0 \\
83,9 \\
- \\
- \\
-\end{array}$ & $\begin{array}{l}-\overline{32,9} \\
891 \\
39,2 \\
103,0 \\
- \\
- \\
-\end{array}$ & $\begin{array}{c}80 \\
27,6 \\
422 \\
24,3 \\
84,8 \\
315 \\
11,7 \\
6,6\end{array}$ & $\begin{array}{c}90 \\
30,0 \\
321 \\
24,7 \\
98,3 \\
352 \\
11,4 \\
7,2\end{array}$ & $\begin{array}{l}100 \\
32,1 \\
251 \\
25,1 \\
112,1 \\
385 \\
10,9 \\
7,6\end{array}$ & $\begin{array}{c}80 \\
23,9 \\
547 \\
23,4 \\
73,2 \\
261 \\
10,2 \\
5,3\end{array}$ & $\begin{array}{c}90 \\
26,1 \\
411 \\
24,0 \\
85,8 \\
298 \\
9,9 \\
5,8\end{array}$ & $\begin{array}{c}100 \\
28,1 \\
319 \\
24,4 \\
98,0 \\
330 \\
9,5 \\
6,2\end{array}$ \\
\hline
\end{tabular}

\section{Le dispositif expérimental} hectare.

Les 6 traitements choisis sont les suivants : les doses sont indiquées en $\mathrm{kg}$ par

1. Témoin

2. $\mathrm{Ca}_{2}: 1500 \mathrm{~kg}$ de $\mathrm{CaO}$ à l'hectare sous forme de calcaire ;

3. $\mathrm{P}: 240 \mathrm{~kg}$ de $\mathrm{P}_{2} \mathrm{O}_{5}$ à l'hectare sous forme de supertriple à $45 \mathrm{p} .100$;

4. $\mathrm{PCa}_{1}: 240 \mathrm{~kg}$ de $\mathrm{P}_{2} \mathrm{O}_{5}$ à l'hectare sous forme de scories à $16 \mathrm{p}$. 100 apportant en plus $675 \mathrm{~kg}$ de $\mathrm{CaO}$ à l'hectare ;

5. $\mathrm{NPCa}_{2} \mathrm{~K}: 200 \mathrm{~kg}$ d'azote sous forme d'ammonitrate à 33 p. 100 ;

$240 \mathrm{~kg}$ de $\mathrm{P}_{2} \mathrm{O}_{5}$ sous forme de scories à 16 p. 100 ;

$1500 \mathrm{~kg}$ de $\mathrm{CaO}$ à l'hectare dont $675 \mathrm{~kg}$ provenant des scories et dont $825 \mathrm{~kg}$ ont été apportés sous forme de calcaire ;

$150 \mathrm{~kg}$ de $\mathrm{K}_{2} \mathrm{O}$ sous forme de sulfate de potassium à 50 p. 100 ;

6. $\mathrm{NPCa}_{1} \mathrm{~K}: 200 \mathrm{~kg}$ d'azote sous forme d'ammonitrate à $33 \mathrm{p} .100$;

$240 \mathrm{~kg}$ de $\mathrm{P}_{2} \mathrm{O}_{5}$ sous forme de scories à 16 p. 100 ;

$675 \mathrm{~kg}$ de $\mathrm{CaO}$ apportés par les scories ;

$150 \mathrm{~kg}$ de $\mathrm{K}_{2} \mathrm{O}$ sous forme de sulfate de potassium à 50 p. 100.

Le phosphore, le calcium et le potassium ont été apportés en surface au cours de l'hiver 1971-1972; l'azote a été apporté en 2 fois :

- $100 \mathrm{~kg}$ au printemps 1972 ,

- $100 \mathrm{~kg}$ au printemps 1973.

Le plan d'expérience est en blocs complets : cinq répétitions (blocs) ont été constituées en tenant compte essentiellement des caractéristiques du peuplement (surface terrière, nombre de tiges, etc...). En effet, cet essai avait été installé principalement pour étudier l'effet de la fertilisation sur la productivité du peuplement forestier. Cependant, pour l'étude mycologique qui nous intéresse ici, nous nous sommes aperçus 
que le type d'humus avait une plus grande influence que la structure du peuplement sur la répartition des espèces de champignons. Nous avons donc redistribué les trente placeaux unitaires de 0,25 ha de superficie en trois groupes en tenant compte uniquement du type d'humus déterminé en cinq points de chaque placeau : mull acide, mull moder et moder. Ainsi pouvons-nous effectuer l'analyse de la variance d'après deux facteurs contrôlés : traitement fertilisant et type d'humus.

\section{Comptage des carpophores}

Les relevés mycologiques dans tous les placeaux ont été réalisés en une seule période, la plus brève possible : du 10 au 14 octobre 1977, soit six années de végétation après l'épandage d'engrais.

Dans chaque placeau, tous les carpophores visibles ont été récoltés, déterminés et comptés après classement par espèce. Les résultats seront donnés en nombre de carpophores par hectare.

Les calculs d'analyse de la variance ont été faits sur la variable transformée $\left(\log _{10}(n+10)\right)$ en raison de sa nature (dénombrement) et du iype de réponse (tout ou rien, pour certaines espèces). Malgré cette transformation, le modèle utilisé n'est pas entièrement satisfaisant. Dans certains cas, nous avons donc considéré comme significatives des différences évidentes que le calcul ne peut pas révéler. Sur les figures des planches no 1 à 4 , un astérisque indique que le calcul a pu mettre en évidence une différence significative au risque de 5 p. 100.

\section{Résultats}

Cinquante-trois espèces ont été relevées au total (tabl. 4), dont quinze sont suffisamment abondantes pour justifier une analyse quantitative des résultats des comptages. Pour les autres, aucune réponse au traitement ou au type d'humus n'apparaît. De toute façon, toute interprétation les concernant serait hasardeuse : l'oubli d'un petit nombre de carpophores biaiserait considérablement les résultats, du fait de leur faible abondance (moins de dix carpophores à l'hectare).

Les résultats des comptages portant sur les quinze espèces retenues sont représentés sur les planches no 1 à 4 . On note d'emblée une très grande variété de comportement en remarquant que toutes les espèces considérées réagissent au moins à l'un des deux facteurs contrôlés : type d'humus ou fertilisation.

\section{Espèces réagissant essentiellement à l'apport de calcaire}

Ces espèces, au nombre de cinq, n'existent jamais dans les placeaux tém oins non fertilisés.

Lycoperdon gemmatum et Lycoperdon piriforme ont un comportement identique. Ils n'apparaissent que sur les traitements $\mathrm{Ca}_{2}$ et presque exclusivement sur les moder. Ces deux champignons atteignent alors une très grande abondance : 4000 carpophores à l'heciare. Une fertilisation complète NPK associée à $\mathrm{Ca}_{2}$ a un effet totalement dépressif. 


\section{TABLEAU 4}

Liste alphabétique des espèces rencontrées

Les espèces mycorhiziennes ou généralement reconnues comme telles sont précédées de $(M)$.

\section{Espèces abondantes}

(M) Amanita citrina, Schaef. ex Fr. Amanite citrine

(M) Boletus chrysenteron, Bull. ex Fr. Bolet à chair jaune Clitocybe rivulosa, Fr. ex Pers.

Collybia butyracea, Bull. ex. Fr. Collybie butyracée

(M) Hebeloma crustuliniforme, Pers. ex Fr. Hébélome échaudé

(M) Laccaria amethystina, Fr. ex Bolt. Laccaire améthyste

(M) Laccaria laccata, Fr. ex Scop. Laccaire laqué

(M) Lactarius blennius, Fr. Lactaire muqueux Lepista inversa, Fr. ex Scop. Clytocibe renversé Lycoperdon gemmatum, Batsch. Vesse de loup perlée Lycoperdon piriforme, Vesse de loup en poire Marasmius dryophilus, Collibie des chênes

Rhodopaxillus nudus, (Bull. ex Fr.) Maire. Tricholome nu, «pied bleu »

(M) Russula fellea, Fr. Russule amère

(M) Russula mairei, Singer

\section{Espèces peu abondantes}

(M) Amanita phalloïdes, Vaill. ex Fr. Amanite phalloïde

(M) Amanita rubescens, Pers. ex Fr. Amanite rougissante, Golmotte

(M) Bolefus subtomentosus, Lin. ex Fr. Bolet subtomenteux

(M) Clavaria cristata Clitocybe ditopa, Fr.

Clitocybe infundibuliformis, Scop. ss Cke. Clitocybe en entonnoir Clitocybe mellea (Fr. ex Fl. Dan.) Rick. Armillaire couleur de miel Clitocybe metachroa, $\mathrm{Fr}$.

Collybia distorta, Fr. Collybie distordue

Collybia platyphylla, Pers. Collybie à lames larges

Collybia radicata, Relh. Collybie radicante

Collybia velutipes, Curt. Collybie à pied velouté

Coprinus atramentarius, Bul. Coprin noir d'encre

Geophila aeruginosa, Lurt. Vert de gris

Geophila fasciculare, Fr. Hypholome en touffe

Geophila sublateritia, Sch. Hypholome briqueté

(M) Hebeloma sp. (indéterminé)

Helvella lacunosa, Helvelle lacuneuse

Hydnum repandum, Hydne bosselé (pied de mouton)

Hydnum rufescens, Hydne roux (pied de mouton)

(M) Inocybe friesii, Heim

(M) Inocybe lanuginosa, Bul.

(M) Lactarius subdulcis, Fr. Lactaire doucâtre

(M) Lactarius vellereus, Fr. Lactaire velouté

Marasmius alliaceus, Fr. ex Jacq. Marasme à odeur d'ail

Mycena pura, Pers. Mycène pur

Pluteus cervinus, Sch. Plutée couleur de cerf

(M) Russula cyanoxantha (Schaef ex Schw.) Fr. Russule charbonnière

(M) Russula grisea, Gil. Russule grise

(M) Russula ochroleuca, Pers. ex Fr. Russule blanc ochracé

(M) Russula nigricans, Bul. Russule noircissante

(M) Russula vesca, Fr. Russule comestible

(M) Tricholoma columbetta, Fr. Tricholome colombette

(M) Tricholoma portentosum, Fr. Tricholome prétentieux

(M) Tricholoma rutilans, Sch. Tricholome rutilant

(M) Tricholoma saponaceum, Fr. Tricholome à odeur de savon

(M) Tricholoma virgatum, Tricholome vergeté 


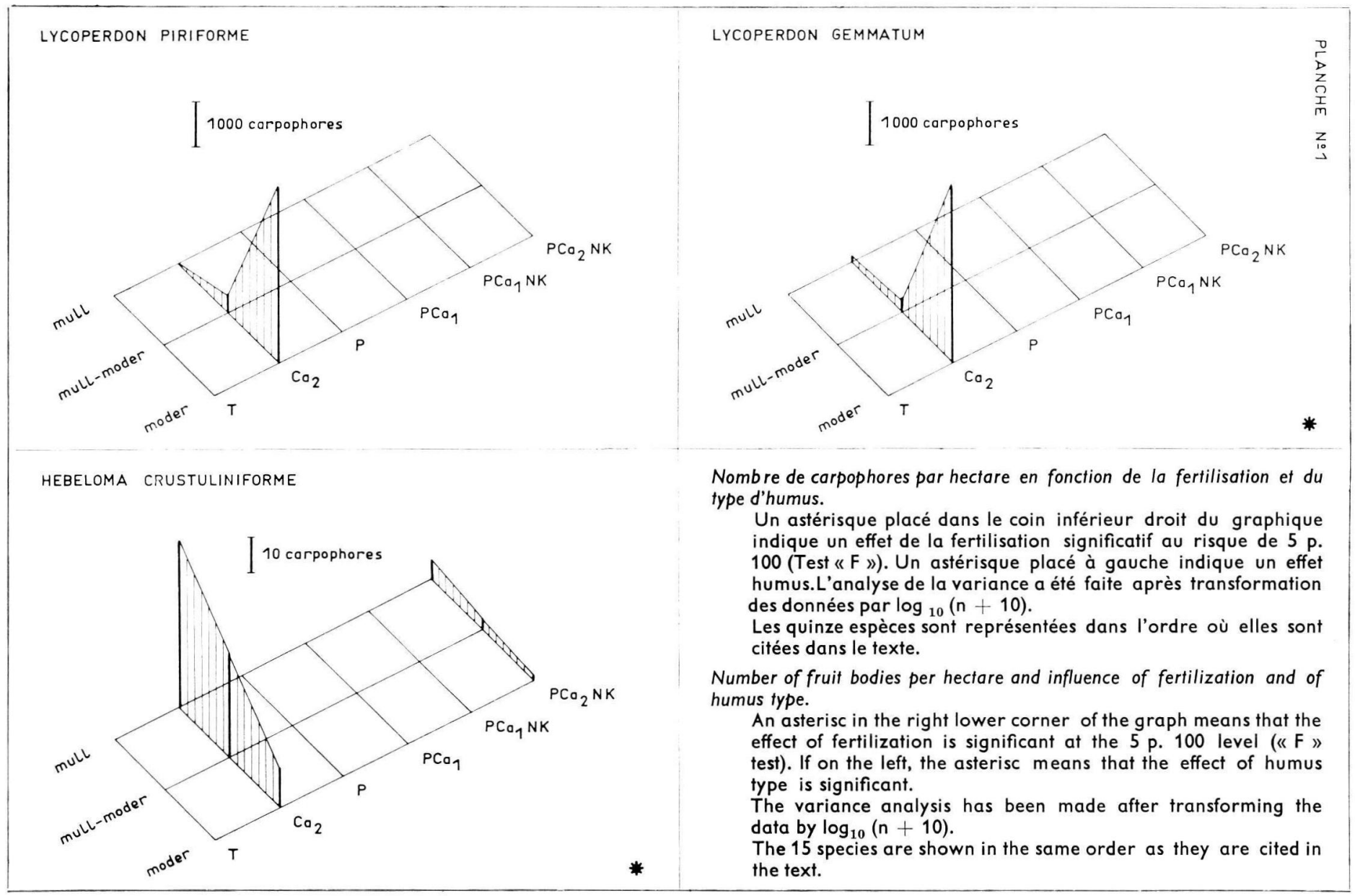




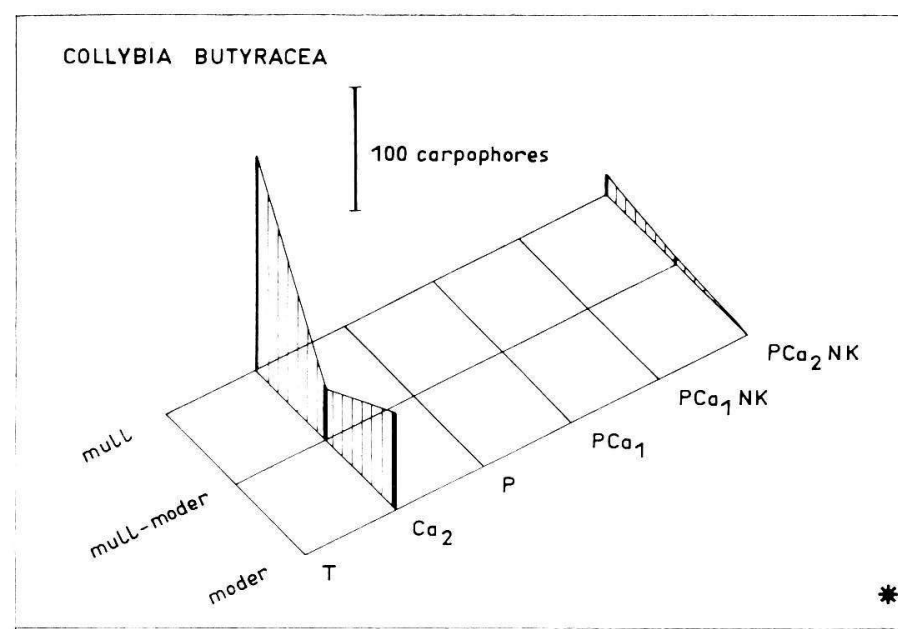

CLITOCYBE RIVULOSA

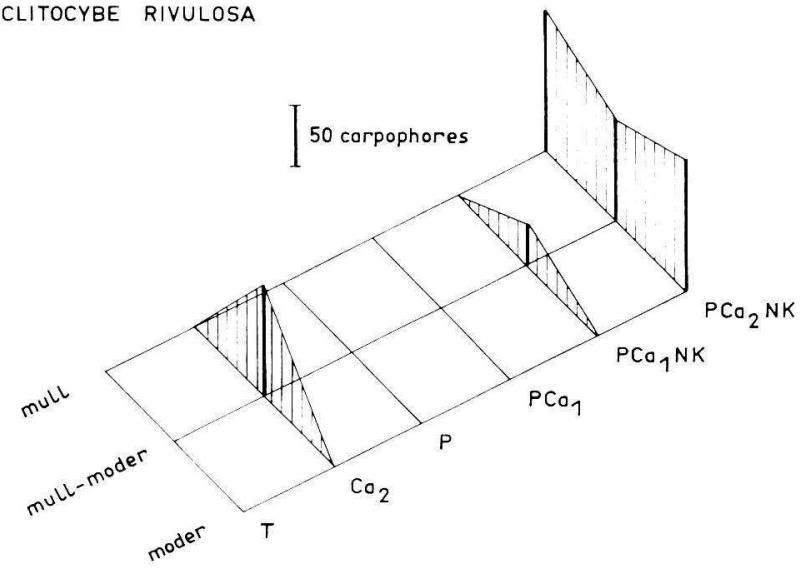

RHODOPAXILLUS NUDUS

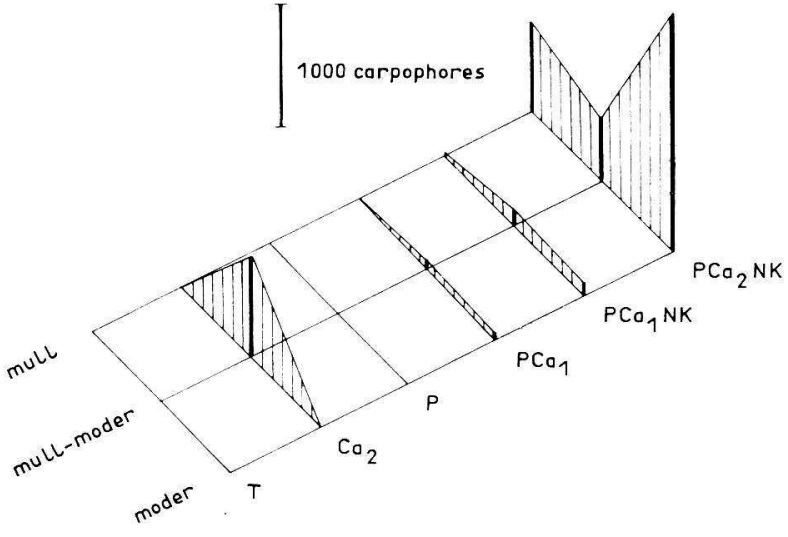




$$
=
$$




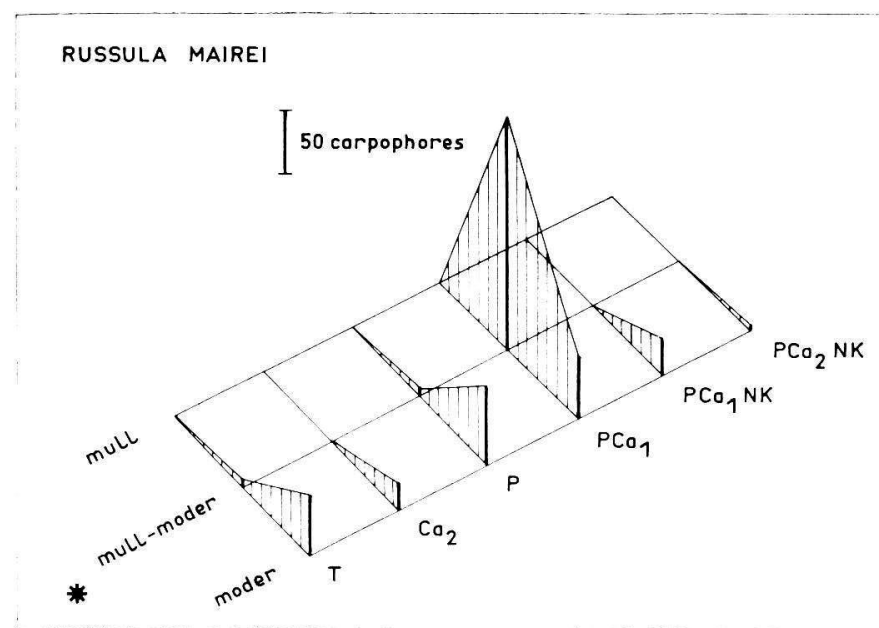

AMANITA CITRINA

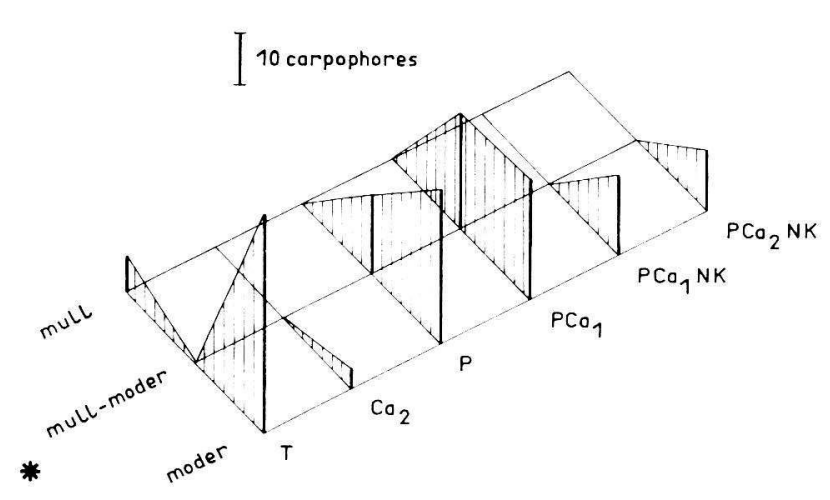

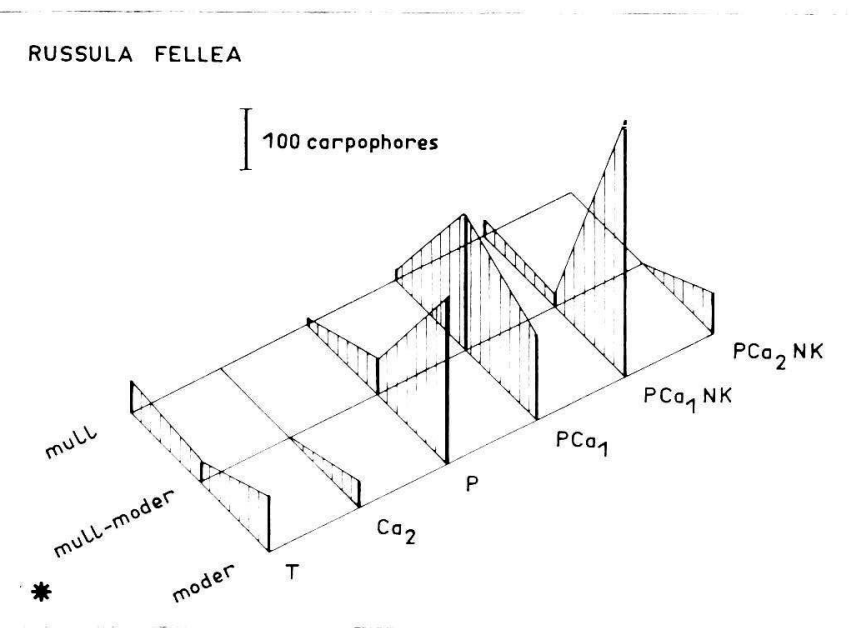

LACTARIUS BLENNIUS

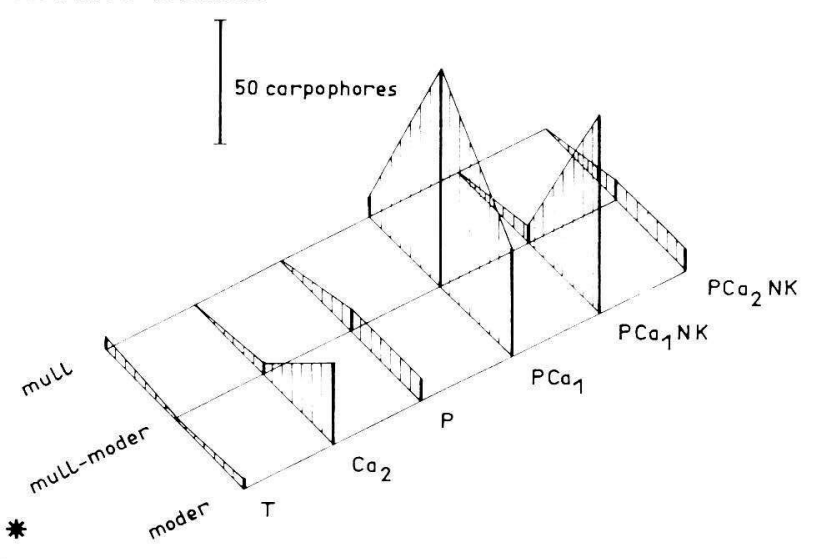

$\overrightarrow{8}$ 
La fructification d'Hebeloma crustuliniforme et de Collybia butyracea est également induite par l'apport de calcaire. L'association de NPK à $\mathrm{Ca}_{2}$ a aussi un effet dépressif, mais on relève encore quelques carpophores dans ce traitement. L'effet du type d'humus se manifeste par une fructification plus abondante sur les mull.

On peut également ranger Laccaria laccata dans le groupe des espèces réagissant à l'apport de calcaire. Cette espèce apparaît dans tous les traitements ayant reçu du calcium, soit sous forme de calcaire, soit sous forme de calcium des scories. La fertilisation complète n'a pas d'effet dépressif.

\section{Espèces réagissant essentiellement à l'apport de phosphore}

Boletus chrysenteron, peu abondant dans le témoin, voit sa fructification favorisée par les traitements « $\mathrm{P}$ seul » et « $P C \mathrm{a}_{1} \mathrm{NK}$. » Cependant, la présence de calcaire accompagné ou non d'azote et de potassium a un effet dépressif.

\section{Espèces réagissant essentiellement à une fertilisation complète (NPKCa)}

L'espèce la plus caractéristique de ce type de comportement est Lepista inversa qui n'apparaît que sur NPCa $\mathrm{K}_{1} \mathrm{~K}$ ef surtout sur $\mathrm{NPCa}_{2} \mathrm{~K}$ (2 000 carpophores à I'hectare). Cette espèce n'apparaît pas sur les mull.

Deux espèces ont un comportement similaire. Rhodopaxillus nudus et Clitocybe rivulosa. Elles réagissent favorablement à une fertilisation complète. Leurs fructifications semblent également induites, mais à un degré moindre, par les traitements comprenant des apports de calcaire ou de calcium. Le « pied bleu », Rhodopaxillus nudus, est particulièrement abondant sur les traitements $\mathrm{NPCa}_{2} \mathrm{~K}$ (2000 carpophores/ha).

Laccaria amethystina, qui existe dans tous les traitements y compris le témoin, est nettement plus abondant dans les deux traitements où la fertilisation est complète.

Quant à Marasmius dryophilus, il réagit de façon exactement contraire : la fertilisation a un effet dépressif. De plus, il est sensible au type d'humus, avec un maximum sur les moder.

\section{Espèces réagissant essentiellement au type d'humus et peu ou pas à la fertilisation}

Nous avons déjà vu que Marasmius dryophilus était plus souvent présent sur les moder que sur les mull. Ce cas se représente plusieurs fois : deux russules (Russula fellea et Russula mairei) présentent un maximum d'abondance sur moder et n'apparaissent que très rarement sur mull.

Cette répartition préférentielle semble également se produire pour certaines autres russules (Russula ochroleuca, Russula nigricans, Russula cyanoxantha, Russula vesca). Le faible nombre de carpophores de ces espèces ne permet cependant pas de tirer des conclusions certaines.

Amanita citrina et Lactarius blennius fructifient plus abondamment sur moder. L'effet de la fertilisation sur la fructification de cette dernière espèce est difficile à interpréter. Les combinaisons $\mathrm{PCa}_{1}, \mathrm{PCa}_{1} \mathrm{NK}$ semblent favorables, tandis que $\mathrm{PCa}_{2} \mathrm{NK}$ aurait un effet dépressif. 


\section{Discussion et conclusion}

Le principe et les conditions de cette étude appellent trois remarques :

- en raison du caractère instantané des complages, nous avons ignoré certaines espèces estivales comme Cantharellus cibarius. D'autre part, l'abondance d'une espèce varie en fonction du temps;

- nous n'avons compté que les carpophores, organes visibles des champignons. Or ceux-ci peuvent être présents sous forme de mycelium végétatif et/ou de mycorhizes lorsque leurs carpophores font défaut. A l'inverse, l'effet d'un traitement qui fait apparaître les carpophores d'une espèce peut être interprété de deux façons : ou bien il induit la fructification d'un mycelium déjà présent, ou bien permet l'installation du champignon dans le sol à partir de spores allogènes, puis sa fructification ;

- les conséquences de l'épandage d'engrais en forêt sont complexes. Par exemple, celles d'un apport de calcaire broyé ne se limitent pas à une simple augmentation du $\mathrm{pH}$ ou de la teneur en calcium échangeable des horizons supérieurs : la minéralisation de la matière organique et de l'azote est modifiée par l'évolution de la microflore et de la microfaune; finalement, l'écosystème tout entier est plus ou moins perturbé.

II est donc nécessaire d'être prudent dans les conclusions relatives aux exigences trophiques des champignons que nous avons étudiés.

Ces restrictions étant posées, plusieurs conclusions intéressantes peuvent être tirées :

- globalement, la fertilisation, même sous forme d'un apport de calcaire seul, enrichit considérablement la macroflore fongique, ainsi que le nombre de carpophores formés.

C'est ainsi que huit espèces, absentes sur les placeaux témoins, ont fructifié après fertilisation. Il s'agit de Lycoperdon gemmatum et L. piriforme, Hebeloma crustuliniforme, Collybia butyracea, Rhodopaxillus nudus, Laccaria laccata, Lepista inversa, ef Clitocybe rivulosa.

- Il n'est pas possible de distinguer les espèces saprophytes des espèces mycorhiziennes du point de vue de leur comportement vis-à-vis du type d'humus, et surtout des traitements. Par exemple, Hebeloma crustuliniforme, mycorhizien, réngit de la même façon que Collybia butyracea, saprophyte follicole.

- Les huit espèces nouvelles qui ont fructifié après fertilisation sont toutes des espèces caractéristiques des mull mésotrophes. Parmi ces huit espèces, quatre réagissent uniquement à un apport de calcium, et trois (Lepista inversa, Rhodopaxillus nudus et Clitocybe rivulosa) a un apport de calcium doublé d'un niveau de fertilité élevé.

Il est assez étonnant de noter que trois de ces espèces du mull mésotrophe ( $L y c o-$ perdon piriforme et L. gemmatum, Lepista inversa) fructifient après fertilisation, mais uniquement sur moder et mull-moder, et non sur mull acide.

Parmi les espèces acidiphiles normalement présentes dans le dispositif, soit sur mull acide, soit sur moder, aucune n'a disparu du fait de la fertilisation. Seul Marasmius dryophilus a une fructification nettement diminuée par un niveau de fertilité élevé. Certaines, au contraire, fructifient plus abondamment après fertilisation, comme Boletus chrysenteron qui est très sensible à la fertilisation phosphatée. 
- Enfin, pour l'une des espèces présentes, la fertilisation semble présenter un intérêt économique : Rhodopaxillus nudus (Tricholome nu, pied bleu, pied violet) est un bon comestible. Il est totalement absent dans les placeaux témoins, mais on récolte 2000 carpophores à l'hectare sur le traitement $\mathrm{PCa}_{2} \mathrm{NK}$, soit $21 \mathrm{~kg}$ à l'hectare pour la seule récolte du 14 octobre 1977. Nous avons effectué deux nouvelles récoltes après repousse, les 26 octobre et 16 novembre 1977 , avec respectivement 17 et $20 \mathrm{~kg}$ par hectare, ce qui fait un total de $58 \mathrm{~kg} / \mathrm{ha}$. Comme ce champignon peut fructifier pendant presque toute la saison de végétation, à la suite de périodes de refroidissement relatif (Romagnesi, 1967), il n'est pas déraisonnabie d'espérer une production d'au moins $70 \mathrm{~kg} / \mathrm{ha}$. Pendant l'automne 1977, le pied bleu se vendait $30 \mathrm{~F} \mathrm{le} \mathrm{kg}$ sur le marché de Nancy. La valeur de la récolte annuelle peut donc être estimée à $2100 \mathrm{~F}$ par hectare, ce qui, grossièrement et compte tenu du transport et du temps de ramassage, doit représenter sur le terrain entre 1000 et $1500 \mathrm{~F}$.

Parallèlement, on peut calculer la valeur du bois produit annuellement par le peuplement. Cette hêtraie, de classe I dans la table de production de Schober (1972), produit actuellement $11 \mathrm{~m}^{3} /$ ha/an (accroissement courant à 90 ans). Le prix moyen sur pied du Hêtre dans le Nord-Est était de $100 \mathrm{~F}$ lors des ventes de l'automne 1976 (Office National des Forêts, 1977), ce qui donne pour ce peuplement $1100 \mathrm{~F} / \mathrm{ha} / \mathrm{an}$ environ.

On voit que la valeur des « pieds bleus » produits annuellement est du même ordre de grandeur que celle du bois (nous ne tenons pas compte de l'augmentation de production de bois éventuellement apportée par la même fertilisation $\mathrm{PCa}_{2} \mathrm{NK}$ : elle est en effet très faible, sinon nulle, jusqu'à présent). Le coût de la fertilisation $\mathrm{PCa}_{2} \mathrm{NK}$ est évalué à $2500 \mathrm{~F} /$ ha (engrais + épandage) (prix 1977). II faut donc seulement deux ou trois ans pour que la valeur des champignons ainsi produits permette de récupérer l'investissement. Or, cette production de «pieds bleus » a été évaluée six ans après l'épandage des engrais, et nous avons la preuve qu'elle dure depuis au moins trois ans (bien que les conditions climatiques aient été très différentes pendant ces années). Il est vraisemblable qu'elle durera encore plusieurs années. II n'est donc pas impossible d'envisager l'application de cette technique de production de champignons comestibles, qui permettrait de valoriser à très court terme de nombreuses forêts. En effet, les observations réalisées à Darney ne constituent pas un cas exceptionnel : une reconnaissance très rapide réalisée en automne 1977 dans deux autres essais de fertilisation montre le même effet très positif des traitements complets sur la fructification de Rhodopaxillus nudus, alors que cette même espèce est absente sur les témoins et très rare dans la région. Il s'agit d'essais dans le Limousin (épicéas sur granite à moyenne altitude) et en Bretagne (hêtres sur limon très acide).

Notons cependant qu'en France des tentatives de culture de Rhodopaxillus nudus sur litières n'ont pas eu de suite en l'absence de marché pour cette espèce. D'autre part, certaines années, les conditions climatiques sont telles que la fructification de cette espèce est nulle ou très faible.

II n'est pas interdit de penser que la fertilisation puisse favoriser la fructification d'autres champignons comestibles très recherchés, dans diverses conditions écologiques. 


\section{Remerciements}

Nous tenons à remercier la Société des Scories Thomas, qui a participé financièrement à l'installation du dispositif, et Messieurs Barbas (Directeur d'Ecole) et Guinberteau (INRA, Bordeaux) qui ont bien voulu nous apporter leur aide pour la détermination de certains champignons.

\section{Summary \\ Mineral fertilization and fructification of higher fungi in a beech forest}

All the sporophores of higher fungi were counted in a fertilization trial in October 1977 in a 90 year old beech stand (Fagus silvatica) in the Lower Vosges region. The soil has developed on Voltzia sandstone (Lower trias) and it ranges from brown acid soil with acid mull to cryptopodzolic soil with moder.

Among the fifty three species recorded, fifteen were abundant. Four groups may be described according to fertilization and humus type :

- species mainly influenced by liming (type : Lycoperdon gemmatum, only found on plots with a high amount of liming alone) ;

- species mainly influenced by phosphorus (type : Boletus chrysenteron, whose fructification is enhanced by phosphorus and inhibited by liming) ;

- species mainly influenced by complete NPKCa fertilization type : Lepista inversa which only occurs on the complete treatments);

- species mainly influenced by the humus type (type : Russula fellea, which occurs only on mullmoder and moder, never on mull).

Fertilization is likely to prove economically interesting for one edible species, Rhodopaxillus nudus (Amethyst Agaric, wood blewits). It is totally missing on the control plots, whereas on plots with complete NPKCa fertilization, a total of $50 \mathrm{~kg}$ per hectare was collected in a month's time. A quick calculation shows that the value of the fungi produced in a year's time is at least equivalent to that of the wood and that it can compensate the costs of fertilization in 2 or 3 years.

\section{Références bibliographiques}

AZEMA R. C., 1973. Essai d'étude écologique des champignons de la région Languedoc-Roussillon (zone méditerranéenne). Docum. myc., Lille, 8-13-36.

CARBIENER R., OURISSON N., BERNARD A., 1972, 1973, 1974 . Premières notes sur les relations entre la répartition des champignons supérieurs et celle des groupements végétaux dans les forêts de la plaine d'Alsace entre Strasbourg et Sélestat. Bulletin de la Société d'Histoire naturelle de Colmar, $55^{\mathrm{e}}$ vol.

CHEVASSUT G., MOUSAIN D., 1973. La macroflore fongique du Pin maritime : essai d'analyse mycosociologique de deux stations de Pin maritime dans la région de Montpellier. Bulletin de la Société mycologique de France, extrait du tome LXXXIX, no 3-4, p. 229.

DARIMONT F., 1973. Recherches mycosociologiques dans les forêts de Haute-Belgique. Institut Royal des Sciences naturelles de Belgique. Mémoire no 170, 2 tomes, 219 p., fig. illust.

EINHELLINGER A., 1964. Die Pilze der Eichen-Mainbuchenwälder des Münchner Lohwaldgürtels. Ber. Bay. Bot. Ges., 37, 11-30.

JAHN H., NESPIAK A., TÜXEN R., 1967. Pilzsoziologische Untersuchungen in Büchenwaldern (Carici-Fagetum, Melico-Fagetum und Luzula-Fagetum) des Wesergebirges. Mitteil. Florist-Soziolog. Arbeitsgemeinschaft Rinteln N.F., 11-12, 159-167.

KRAFT M. M., 1956. Sur la répartition d'Amanita caesarea (Fr. ex. Scop.) Bulletin de la Société botanique suisse, tome $66,39-61$.

KRAFT M. M., 1958. Sur la répartition d'Hygrophorus marzuolus (Fr.). Bres. Bulletin de la Société botanique suisse, tome $68,254-288$.

KÜHNER R., ROMAGNESI H., 1963. Flore analytique des champignons supérieurs. Masson ef Cie, Paris, 556 p.

OFFICE NATIONAL DES FORETS., 1977. Les grandes ventes d'automne 1976 dans les forêts soumises au régime forestier. R.F.F., XXIX, 2.

ROMAGNESI H., 1967. Nouvel atlas des champignons. Bordas, 4 vol. 